\title{
Review Article \\ The Size of the Human Proteome: The Width and Depth
}

\author{
Elena A. Ponomarenko, Ekaterina V. Poverennaya, \\ Ekaterina V. Ilgisonis, Mikhail A. Pyatnitskiy, Arthur T. Kopylov, \\ Victor G. Zgoda, Andrey V. Lisitsa, and Alexander I. Archakov
}

Institute of Biomedical Chemistry, Moscow 119121, Russia

Correspondence should be addressed to Alexander I. Archakov; alexander.archakov@ibmc.msk.ru

Received 18 January 2016; Revised 11 April 2016; Accepted 19 April 2016

Academic Editor: Frantisek Foret

Copyright (C) 2016 Elena A. Ponomarenko et al. This is an open access article distributed under the Creative Commons Attribution License, which permits unrestricted use, distribution, and reproduction in any medium, provided the original work is properly cited.

\begin{abstract}
This work discusses bioinformatics and experimental approaches to explore the human proteome, a constellation of proteins expressed in different tissues and organs. As the human proteome is not a static entity, it seems necessary to estimate the number of different protein species (proteoforms) and measure the number of copies of the same protein in a specific tissue. Here, metaanalysis of neXtProt knowledge base is proposed for theoretical prediction of the number of different proteoforms that arise from alternative splicing (AS), single amino acid polymorphisms (SAPs), and posttranslational modifications (PTMs). Three possible cases are considered: (1) PTMs and SAPs appear exclusively in the canonical sequences of proteins, but not in splice variants; (2) PTMs and SAPs can occur in both proteins encoded by canonical sequences and in splice variants; (3) all modification types (AS, SAP, and PTM) occur as independent events. Experimental validation of proteoforms is limited by the analytical sensitivity of proteomic technology. A bell-shaped distribution histogram was generated for proteins encoded by a single chromosome, with the estimation of copy numbers in plasma, liver, and HepG2 cell line. The proposed metabioinformatics approaches can be used for estimation of the number of different proteoforms for any group of protein-coding genes.
\end{abstract}

\section{From Human Genome to Human Proteome}

Genome sequencing [1] deciphered the number of proteincoding genes, establishing an initial estimation of complexity associated with human molecular biology. The next step is to obtain similar benchmarks at the proteome level. Two recent articles described creation of a draft of the human proteome $[2,3]$. Nevertheless, considerable efforts are still required for exploring the space (or size) of the human proteome, as a compulsory constellation of molecular profiles of different tissues and organs. The human proteome is quite a dynamic entity [4] and this property should be considered in two dimensions. The first is to estimate the number of different protein types (proteome width), as well as measure protein copies number in particular tissues (proteome depth).

Following the hypothesis of "one gene = one protein," there should be at least $\sim 20,000$ nonmodified (canonical) human proteins. Taking into account products of alternative splicing (AS), those containing single amino acid polymorphisms (SAPs) arising from nonsynonymous singlenucleotide polymorphisms (nsSNPs), and those that undergo PTMs $[4,5]$, as many as 100 different proteins can potentially be produced from a single gene. Of the many different terms proposed to describe protein variants [6], here, we chose "protein species" [7] or "proteoforms" [6].

Experimental validation of protein species is limited by the analytical sensitivity of proteomic technology. This means that the sensitivity of the technology determines the ability to detect rare protein species. This limitation originates from the basic difference between genomics and proteomics [8]. Genomics relies upon PCR [9] to amplify DNA or RNA molecules in a biological sample to concentrations above the detection threshold. However, there currently exists no comparable high-throughput technology capable of multiplying the copies of a single protein [8].

The $100 \%$ coverage of protein sequence using bottomup MS is not attainable; thus, it is impossible to detect all potential protein species expressed from the same gene. 
Generally, proteome investigations are focused on the master proteins resembling at least one of the many possible proteoforms, coded by the gene and containing at least one MS-detectable proteotypic peptide. The sequence could be modified or nonmodified, so this means that the master protein could be present as a single protein or as a set of proteins. Master proteome of a single chromosome is the result of the identification and measurement of all master proteins encoded by the chromosome and expressed in the selected type of biological material. For experimental validation of proteoforms, the targeted MS analysis should be performed in order to probe candidate sequence alteration. The bioinformatic analysis of the diversity of protein species was anticipated to create the backbone for the future experimental exploration of the proteome space.

\section{How Many Different Proteins Are Necessary to Support Human Function?}

The number of different proteins comprising the human proteome is a core proteomics issue. Researchers propose numbers between 10,000 [10] and several billion [6] different protein species. Here, we describe the theoretical prediction for the number of different proteoforms that might arise from AS, SAP, or PTM events.

The data was derived from neXtProt, which contains only human proteins and their modifications and sequence features [11]. The neXtProt annotation of AS, SAP, and PTM originated from biocuration of the data from repositories, literature, and prediction tools. Information on possible protein sequence variability is represented as the number of AS variants, nsSNPs/SAPs, and PTMs per gene.

Our assumption was that database extension and annotation are a constituent process, whose rate is mostly limited by the number of the researchers and annotators around the world. The rate is slightly dependent on the capacity of communication channel and information accessibility, as these were not changed too much for 10-15 years for the needs of PubMed or UniProt users. Therefore, the extension of the number of annotations in a certain database would generally be affected by the technology achievements, gained by increasing the sensitivity/throughput of the bioanalytical method.

From the above, we proposed that the volume of representative data uploaded to UniProt [12] each year from 2005 was sufficient to calculate the average number of protein variants per one gene and the numbers for each type of variation. Interestingly, since 2010, the average number of modifications per one gene has remained nearly the same, despite the continuous increase in reviewed annotations. The average number of modifications specifically by AS (40\% reviewed annotations out of all data records), SAP (60\% reviewed annotations), or PTM (37\%) remains almost unchanged.

The saturation in the number of annotations for genomedependent SAPs, transcription-dependent ASs, and posttranslational-dependent PTMs is quite remarkable. While PTM determination depends upon the sensitivity of protein analytics, SAP and AS detection have virtually no limitations in sensitivity and are actively accumulated via large-scale projects [13]. Despite such differences, all of the technologies have synchronously acquired saturation levels, indicating balance between data derived from using standard proteinchemistry techniques (accumulated over the last 50 years) and data derived from high-throughput next-generation sequencing (NGS).

For estimating the potential number of proteins, three different cases of combination of PTM, SAP, and AS events were considered (see (1)-(3)). Combinatorial variations were ignored, since there are no systematic experimental data describing the cooccurrence of various modification types in the protein species. This is just one of the possible ways for solving the problem of how to estimate a potential number of proteins based on the data of protein variance that has already been accumulated on the postgenomic knowledge bases. Equation (1) assumes that PTMs appear exclusively in the canonical sequences of proteins, but not in splice variants. Equation (2) assumes that PTMs and SAPs can occur both in proteins encoded by canonical sequences and in splice variants. Equation (3) assumes that all modification types (AS, SAP, and PTM) occur independently. Hence,

$$
\begin{aligned}
& \text { Nps }=N *(\text { ASav }+ \text { SAPav }+ \text { PTMav }) \\
& \text { Nps }=(N+\text { AS }) *(\text { SAPav }+ \text { PTMav }) \\
& \text { Nps }=N * \text { ASav } * \text { SAPav } * \text { PTMav, }
\end{aligned}
$$

where Nps represents the number of protein species, $N$ represents the total number of protein encoding genes, AS is the number of species produced by alternative splicing, ASav is the average number of splice variants per one protein encoding genes, SAPav is the average number of nsSNPs, and PTMav is the average number of PTM events per one protein encoding gene.

Generally, SAPs are predetermined at the DNA level, and AS arises from modifications at the mRNA level, while PTMs occur at the protein level. These three processes cannot be viewed as independent events, given that there is an intrinsic relationship between the processes of gene expression, transcription, and translation, aimed at regulating and preserving a cell. Furthermore, enriching MS/MS searches through a database containing all possible combinations of protein variations would lead to combinatorial collapse, despite the type of approach used [14].

The neXtProt (ver. 2015_06) search for protein AS modifications revealed 21,921 AS variants in 10,519 proteincoding genes ( $2.1 \pm 0.1$ variants/gene, including one canonical sequence). The greatest number of modified forms $(434,398$, without cancer-related items derived from the COSMIC cancer mutation database [15]) was due to the emergence of SAPs resulting from nsSNPs in 18,986 protein-coding genes $(22.1 \pm 3.9$ variants/gene). PTMs added $6.6 \pm 0.8$ modified proteins/gene (94,036 PTMs in 14,006 protein-coding genes). Applying these numbers to the equations $(N=20,043)$, we estimate that in humans there exist 0.62 or 0.88 or 6.13 million protein species.

The above results were matched to the data on AS- and SAP-derived variances obtained from our NGS results of liver 
tissue transcriptome profiling [16-18]. According to NGS results, the average number of detected splice variants was 1.3 per protein-coding gene (or 2.3 per gene including canonical variant), which is comparable to neXtProt data. The average number of SAP-containing proteoforms was $\sim 1.4$ per one gene, so much lower than that calculated from neXtProt data. These differences relate to the fact that neXtProt provides information from many different experiments ("aggregate human population"), while specific NGS data indicates SAP events for an individual sample or tissue (individual variances).

As proteomic knowledge bases consolidate information regarding protein variability in the human population, several million different proteins will ultimately populate the "aggregated" human proteome. To decipher variability inherent in predicting proteome space for an individual, more precise estimation of the numbers of AS- and SAP-contained proteins can be achieved using results of transcriptome profiling of specific tissue samples.

\section{How Many Protein Species Are Detectable Today?}

According to the Plasma Proteome Database (ver. 06_2015) [19], 10.5 thousand blood-plasma proteins have been detected and less than $10 \%$ (1278 of 20,043 human proteins) have been measured in a quantitative manner. The primary issue concerning experimental validation of existing sets of theoretically predicted proteins is the limit of analytical sensitivity of proteomic technology. Analytical sensitivity is determined by instrument-dependent detection limit and biomaterialdependent dynamic protein concentration ranges. Blood plasma is a complex mixture with a dynamic range of protein concentrations varying by $>10$ orders of magnitude [20], while the protein concentration range of tissue or cell lines is within seven orders of magnitude [21]. The challenge is in detecting low- and ultralow-abundance species with concentrations $<10^{-12} \mathrm{M}$ in the presence of high-copied protein molecules at concentrations $>10^{-6} \mathrm{M}$ [22].

Assuming the ultrasensitive capacity of oligonucleotide analytics, it is instructive to consider that transcriptome research results are often determined based on copies of RNA molecules rather than concentrations [23]. Operating at low- $\left(<10^{-12} \mathrm{M}\right)$ and ultralow $\left(<10^{-15} \mathrm{M}\right)$ concentrations of proteins implies that quantifying protein in copy numbers rather than in concentration units enables comparison of transcriptomic and proteomic results [24].

Proteins are commonly quantified in the proteomics field [25] by the concentration in the biological sample, $C$, reported as mol/L (molarity, $\mathrm{M}$ ). The corresponding number of protein copies, $N$, in $1 \mathrm{~L}$ can be calculated out of concentration units as follows:

$$
\begin{aligned}
& N=\left(\frac{C * V}{R_{\mathrm{A}}}\right), \\
& C=\left(\frac{m}{M_{w} * V}\right),
\end{aligned}
$$

where $R_{A}$ represents the reverse Avogadro's number, $10^{-24} \mathrm{M}$ [26], $V$ represents the sample volume, $m$ represents the protein content, and $M_{w}$ represents molecular weight of the protein.

Formulas (4) address the major challenge of proteomics: shift from concept of the concentration units to counting single biomacromolecules in a sample (tissue) [27].

The triple-quadrupole mass spectrometer makes it possible to achieve $10^{-14} \mathrm{M}[28,29]$ sensitivity for targeted proteins [30]. The sensitivity of SRM protein detection can be further increased up to $10^{-16} \mathrm{M}$ by irreversible chemical binding proteins from large volumes of biological samples [31] (it is not intended to state that all proteins measured were determined with such sensitivity; results of measurements can vary by several orders of magnitude due to different physicochemical properties of proteotypic peptides).

In the context of proteome width, the targeted approach is limited by the need to measure only proteoforms exhibiting a priori assumption of proteotypic peptides, which correctly resemble PTM, SAP, or AS events. In contrast to shotgun MS, SRM cannot discover new, unexpected protein species [32]. Possibilities of top-down and bottom-up MS approaches to address the microheterogeneity of the human proteome were described earlier [33]. Targeted SRM is readily available for detecting SAPs in association with disease, including obesity/diabetes [34] and cancer [35]. For example, SRM/MRM method was applied to measure the quantities of splice forms: three isoforms for transforming growth factor were measured by SRM at concentration level of $10^{-11} \mathrm{M}$ in mouse plasma and human saliva [36]. Another example, osteopontin isoforms, was measured using the SRM assay and revealed that level of isoform was significantly higher for non-small cell lung carcinoma compared with the control group $\left(7 * 10^{-10}\right.$ versus $\left.30 * 10^{-10} \mathrm{M}\right)$ [37]. The application of targeted MS for the detection of PTMs was illustrated for protein glycosylation: $\mathrm{N}$-glycosides were detected in human plasma at a sensitivity level of $10^{-11} \mathrm{M}$ [38] and ubiquitination [39]. From these pilot studies, it follows that the vast majority of predictable proteoforms seem to be present in the concentrations below limit of detection. Further increase in sensitivity of analytical methods is important to uncover diagnostically relevant proteoforms in human biosamples.

Since it was shown that the set of proteins encoded by any human chromosome constitutes a representative portion for the whole human proteome [40], high-, medium-, and lowcopied protein species can be evaluated by sampling master proteins encoded by a single chromosome. As an example of a chromosome-centric proteomic map, we uploaded data from PASSEL [41] (PASSEL IDs: PASS00278, PASS00276, PASS00092, and PASS00742) obtained for master proteins encoded by chromosome 18 [16, 17]. These proteins were measured in three types of biomaterial, including human plasma, liver samples, and HepG2 cells. The measurements were conducted according to Tier 3 (exploratory studies) guidelines [42] using the double targeted strategy, which combines chromosome-centric approach with bottom-up SRM mass spectrometry [43]. 


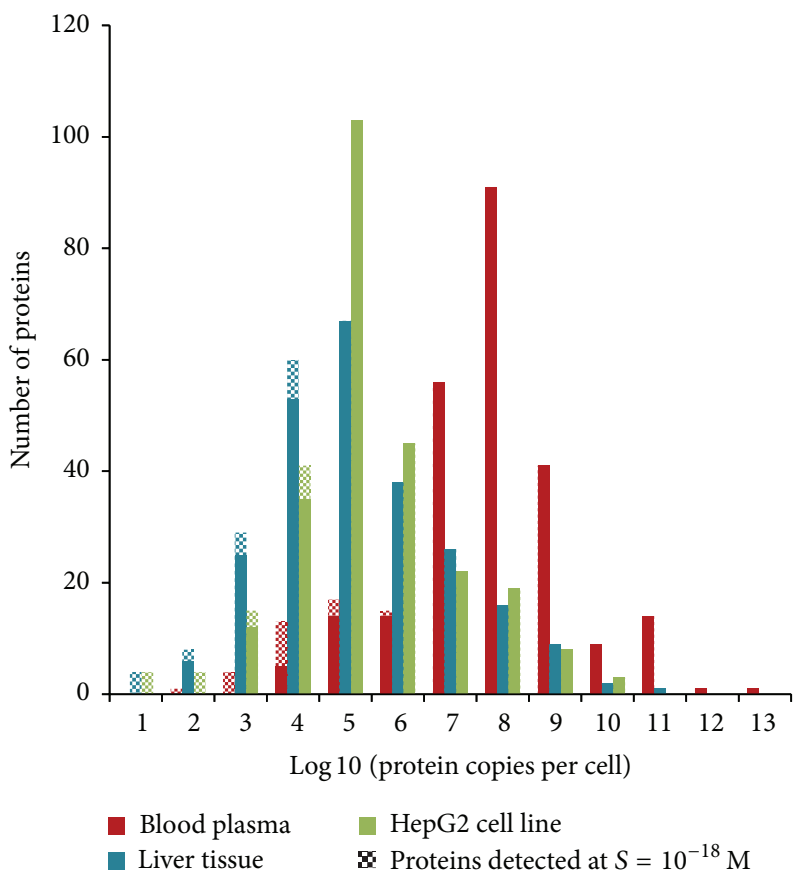

(a)

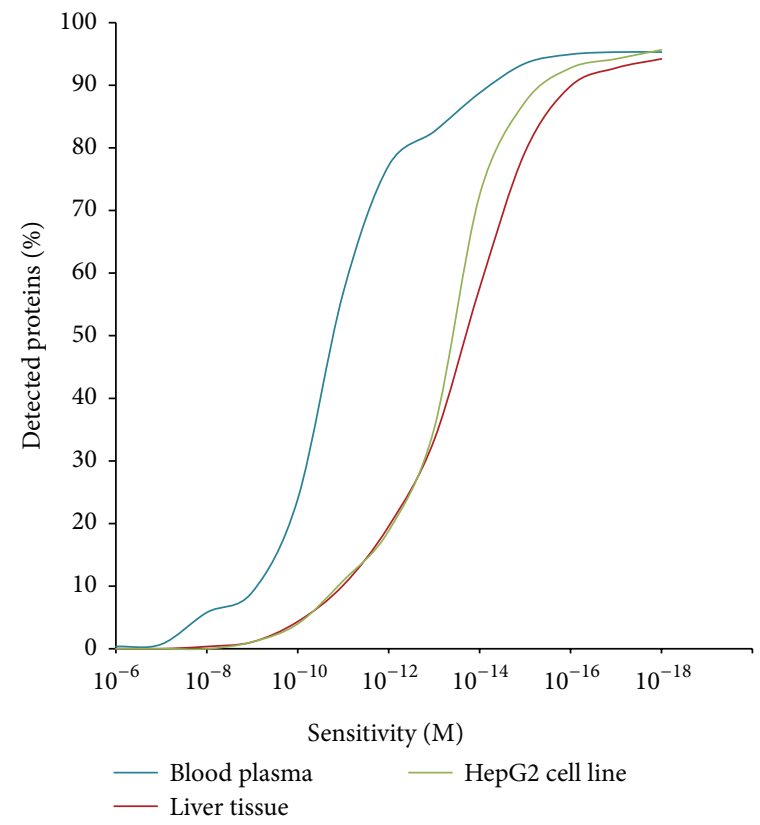

(b)

FIGURE 1: (a) Distribution of the copy numbers of master proteins of chromosome 18 normalized per single HepG2/liver cell or $1 \mu \mathrm{L}$ of plasma. (b) Share as a function of the detected proteins (in \% to the total number of chromosome 18-coded proteins) and the analytical sensitivity.

A bell-shaped distribution histogram for master proteins encoded by chromosome 18 was observed (Figure 1(a)) revealing median of $10^{8}$ copies per $1 \mu \mathrm{L}$ of blood plasma and $10^{5}$ copies per liver/HepG 2 cell. The ascending portion of the curve reflects high- and medium-copied proteins, whereas the descending portion may be explained by either diminished proteome diversity in a biological sample or more probably the notion that the proteins cannot be detected due to low sensitivity of the analytical methods [44]. Interestingly, after increasing the sensitivity of the analytical method from $10^{-14} \mathrm{M}$ to $10^{-18} \mathrm{M}$ by irreversible binding of analytes [30], 14 additional low-copied protein species $\left(<10^{5}\right.$ copies per cell or per $1 \mu \mathrm{L}$ of blood plasma) were gained and quantitatively measured, with at least two proteotypic peptides in each type of biomaterial (see shaded areas in Figure 1(a)). According to the results, there are much more high-abundant protein species in the plasma as compared with the liver or HepG2 cells. It is, therefore, likely that the difficulty in identifying ultralow-copied proteins in plasma is related to the high dynamic concentration range of plasma proteins [22].

To demonstrate the proteome depth, the number of copies of a master protein in a biosample was plotted depending on the sensitivity of proteomic technology (Figure 1(b)). The proteome coverage was expressed as percent share of detected proteins to the total number of chromosome 18 genes, which was 276 according to neXtProt data. As shown in Figure 1(b), the distribution curve for the plasma proteins shifts left relative to the curves for the cells. The total number of detected protein species in liver and HepG2 cells increased relative to human blood plasma.
Future successes in human proteome exploration depend upon the ability to use bioinformatics methods to elucidate existing protein species and targeted MS analysis, highthroughput measurement, and high-performance algorithms for de novo assembly of protein sequences based on MS results. Furthermore, increasing the sensitivity of analytical technology will enable greater access to ultralow-copied proteins and expand opportunities for detection and analysis. In this context, theoretical prediction of the number of proteoforms (estimation of proteome width) and their distribution across the dynamic range (i.e., proteome depth) is ultimately required for planning the workload for the chromosomecentric Human Proteome Project.

\section{Abbreviations \\ AS: $\quad$ Alternative splicing \\ NGS: Next-generation sequencing \\ nsSNPs: Nonsynonymous single-nucleotide polymorphisms \\ SAP: Single amino acid polymorphism.}

\section{Competing Interests}

The authors declare no competing interests.

\section{Acknowledgments}

This work was supported by RSF Grant no. 15-15-30041. 


\section{References}

[1] F. S. Collins, E. S. Lander, J. Rogers, and R. H. Waterston, "Finishing the euchromatic sequence of the human genome," Nature, vol. 50, pp. 162-168, 2005.

[2] M. Wilhelm, J. Schlegl, H. Hahne et al., "Mass-spectrometrybased draft of the human proteome," Nature, vol. 509, no. 7502, pp. 582-587, 2014.

[3] M.-S. Kim, S. M. Pinto, D. Getnet et al., "A draft map of the human proteome," Nature, vol. 509, no. 7502, pp. 575-581, 2014.

[4] C. Karlsson, L. Malmström, R. Aebersold, and J. Malmström, "Proteome-wide selected reaction monitoring assays for the human pathogen Streptococcus pyogenes," Nature Communications, vol. 3, article 1301, 2012.

[5] M. J. Roth, A. J. Forbes, M. T. Boyne II, Y.-B. Kim, D. E. Robinson, and N. L. Kelleher, "Precise and parallel characterization of coding polymorphisms, alternative splicing, and modifications in human proteins by mass spectrometry," Molecular and Cellular Proteomics, vol. 4, no. 7, pp. 1002-1008, 2005.

[6] L. M. Smith and N. L. Kelleher, "Proteoform: a single term describing protein complexity," Nature Methods, vol. 10, no. 3, pp. 186-187, 2013.

[7] P. Jungblut, B. Thiede, U. Zimny-Arndt et al., "Resolution power of two-dimensional electrophoresis and identification of proteins from gels," Electrophoresis, vol. 17, no. 5, pp. 839-847, 1996.

[8] A. Archakov, V. Zgoda, A. Kopylov et al., "Chromosome-centric approach to overcoming bottlenecks in the Human Proteome Project," Expert Review of Proteomics, vol. 9, no. 6, pp. 667-676, 2012.

[9] R. K. Saiki, D. H. Gelfand, S. Stoffel et al., "Primer-directed enzymatic amplification of DNA with a thermostable DNA polymerase," Science, vol. 239, no. 4839, pp. 487-491, 1988.

[10] J. N. Adkins, “Toward a human blood serum proteome: analysis by multidimensional separation coupled with mass spectrometry," Molecular \& Cellular Proteomics, vol. 1, pp. 947-955, 2002.

[11] L. Lane, G. Argoud-Puy, A. Britan et al., "NeXtProt: a knowledge platform for human proteins," Nucleic Acids Research, vol. 40, no. 1, pp. D76-D83, 2012.

[12] R. Apweiler, A. Bairoch, C. H. Wu et al., "UniProt: the universal protein knowledgebase," Nucleic Acids Research, vol. 32, pp. D115-D119, 2004.

[13] G. R. Abecasis, A. Auton, L. D. Brooks et al., "An integrated map of genetic variation from 1,092 human genomes," Nature, vol. 491, pp. 56-65, 2012.

[14] J. S. Cottrell, "Protein identification using MS/MS data," Journal of Proteomics, vol. 74, no. 10, pp. 1842-1851, 2011.

[15] S. A. Forbes, D. Beare, P. Gunasekaran et al., "COSMIC: exploring the world's knowledge of somatic mutations in human cancer," Nucleic Acids Research, vol. 43, no. 1, pp. D805-D811, 2015.

[16] V. G. Zgoda, A. T. Kopylov, O. V. Tikhonova et al., "Chromosome 18 transcriptome profiling and targeted proteome mapping in depleted plasma, liver tissue and HepG2 cells," Journal of Proteome Research, vol. 12, no. 1, pp. 123-134, 2013.

[17] E. A. Ponomarenko, A. T. Kopylov, A. V. Lisitsa et al., "Chromosome 18 transcriptoproteome of liver tissue and HepG2 Cells and targeted proteome mapping in depleted plasma: update 2013," Journal of Proteome Research, vol. 13, no. 1, pp. 183-190, 2014.
[18] A. V. Tyakht, E. N. Ilina, D. G. Alexeev et al., "RNA-Seq gene expression profiling of HepG2 cells: the influence of experimental factors and comparison with liver tissue," $B M C$ Genomics, vol. 15, article 1108, 2014.

[19] B. Muthusamy, G. Hanumanthu, S. Suresh et al., "Plasma proteome database as a resource for proteomics research," Proteomics, vol. 5, no. 13, pp. 3531-3536, 2005.

[20] N. L. Anderson, M. Polanski, R. Pieper et al., "The human plasma proteome," Molecular and Cellular Proteomics, vol. 3, no. 4, pp. 311-326, 2004.

[21] M. Beck, A. Schmidt, J. Malmstroem et al., "The quantitative proteome of a human cell line," Molecular Systems Biology, vol. 7, article 549, 2011.

[22] N. L. Anderson and N. G. Anderson, "The human plasma proteome: history, character, and diagnostic prospects," Molecular \& Cellular Proteomics, vol. 1, no. 11, pp. 845-867, 2002.

[23] R. De Sousa Abreu, L. O. Penalva, E. M. Marcotte, and C. Vogel, "Global signatures of protein and mRNA expression levels," Molecular BioSystems, vol. 5, no. 12, pp. 1512-1526, 2009.

[24] B. Schwanhäusser, D. Busse, N. Li et al., "Global quantification of mammalian gene expression control," Nature, vol. 473, no. 7347, pp. 337-342, 2011.

[25] N. L. Anderson, N. G. Anderson, T. W. Pearson et al., "A human proteome detection and quantitation project," Molecular and Cellular Proteomics, vol. 8, no. 5, pp. 883-886, 2009.

[26] A. I. Archakov, Y. D. Ivanov, A. V. Lisitsa, and V. G. Zgoda, "AFM fishing nanotechnology is the way to reverse the Avogadro number in proteomics," Proteomics, vol. 7, no. 1, pp. 4-9, 2007.

[27] A. V. Lisitsa, "Molar concentration welcomes avogadro in postgenomic analytics," Biochemistry \& Analytical Biochemistry, vol. 04, pp. 4-7, 2015.

[28] R. Kiyonami, A. Schoen, A. Prakash et al., "Increased selectivity, analytical precision, and throughput in targeted proteomics," Molecular \& Cellular Proteomics, vol. 10, no. 2, 2011.

[29] S. Sano, S. Tagami, Y. Hashimoto et al., "Absolute quantitation of low abundance plasma APL1 $\beta$ peptides at Sub-fmol/mL level by SRM/MRM without immunoaffinity enrichment," Journal of Proteome Research, vol. 13, no. 2, pp. 1012-1020, 2014.

[30] A. T. Kopylov, V. G. Zgoda, A. V. Lisitsa, and A. I. Archakov, "Combined use of irreversible binding and MRM technology for low- and ultralow copy-number protein detection and quantitation," Proteomics, vol. 13, no. 5, pp. 727-742, 2013.

[31] A. Archakov, Y. Ivanov, A. Lisitsa, and V. Zgoda, "Biospecific irreversible fishing coupled with atomic force microscopy for detection of extremely low-abundant proteins," Proteomics, vol. 9, no. 5, pp. 1326-1343, 2009.

[32] A. P. Oliveira, C. Ludwig, P. Picotti, M. Kogadeeva, R. Aebersold, and U. Sauer, "Regulation of yeast central metabolism by enzyme phosphorylation," Molecular Systems Biology, vol. 8, article 623, 2012.

[33] A. Lisitsa, S. Moshkovskii, A. Chernobrovkin, E. Ponomarenko, and A. Archakov, "Profiling proteoforms: promising followup of proteomics for biomarker discovery," Expert Review of Proteomics, vol. 11, no. 1, pp. 121-129, 2014.

[34] Z.-D. Su, L. Sun, D.-X. Yu et al., "Quantitative detection of single amino acid polymorphisms by targeted proteomics," Journal of Molecular Cell Biology, vol. 3, no. 5, pp. 309-315, 2011.

[35] Q. Wang, R. Chaerkady, J. Wu et al., "Mutant proteins as cancerspecific biomarkers," Proceedings of the National Academy of Sciences of the United States of America, vol. 108, no. 6, pp. 24442449, 2011. 
[36] X. Liu, Z. Jin, R. O’Brien et al., “Constrained selected reaction monitoring: quantification of selected post-translational modifications and protein isoforms," Methods, vol. 61, no. 3, pp. 304312, 2013.

[37] J. Wu, P. Pungaliya, E. Kraynov, and B. Bates, "Identification and quantification of osteopontin splice variants in the plasma of lung cancer patients using immunoaffinity capture and targeted mass spectrometry," Biomarkers, vol. 17, no. 2, pp. 125-133, 2012.

[38] R. Ossola, R. Schiess, P. Picotti, O. Rinner, R. Reiter, and R. Aebersold, "Biomarker validation in blood specimens by selected reaction monitoring mass spectrometry of $\mathrm{N}$ glycosites," Methods in Molecular Biology, vol. 728, pp. 179-194, 2011.

[39] A. N. Kettenbach, J. Rush, and S. A. Gerber, "Absolute quantification of protein and post-translational modification abundance with stable isotope-labeled synthetic peptides," Nature Protocols, vol. 6, no. 2, pp. 175-186, 2011.

[40] E. Ponomarenko, E. Poverennaya, M. Pyatnitskiy et al., "Comparative ranking of human chromosomes based on postgenomic data," OMICS: A Journal of Integrative Biology, vol. 16, no. 11, pp. 604-611, 2012.

[41] U. Kusebauch, E. W. Deutsch, D. S. Campbell, Z. Sun, T. Farrah, and R. L. Moritz, "Using PeptideAtlas, SRMAtlas, and PASSEL: comprehensive resources for discovery and targeted proteomics," Current Protocols in Bioinformatics, vol. 46, pp. 128, 2014.

[42] S. A. Carr, S. E. Abbatiello, B. L. Ackermann et al., "Targeted peptide measurements in biology and medicine: best practices for mass spectrometry-based assay development using a fit-forpurpose approach," Molecular and Cellular Proteomics, vol. 13, no. 3, pp. 907-917, 2014.

[43] A. Archakov, A. Aseev, V. Bykov et al., "Gene-centric view on the human proteome project: the example of the Russian roadmap for chromosome 18," Proteomics, vol. 11, no. 10, pp. 1853-1856, 2011.

[44] A. V. Lisitsa, E. V. Poverennaya, E. A. Ponomarenko, and A. I. Archakov, "The width of the human plasma proteome compared with a cancer cell line and bacteria," Biomolecular Research \& Therapeutics, vol. 4, article 132, 2015. 

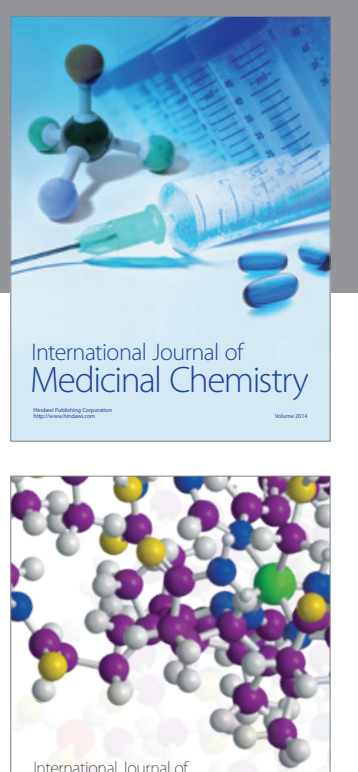

Carbohydrate Chemistry

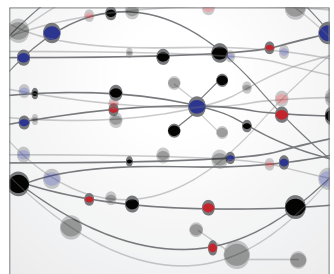

The Scientific World Journal
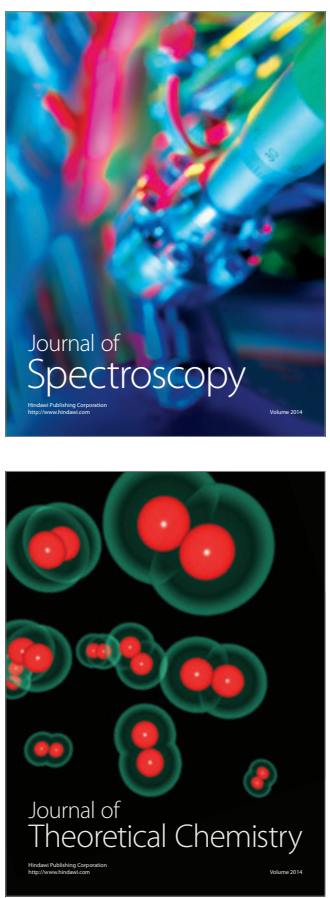
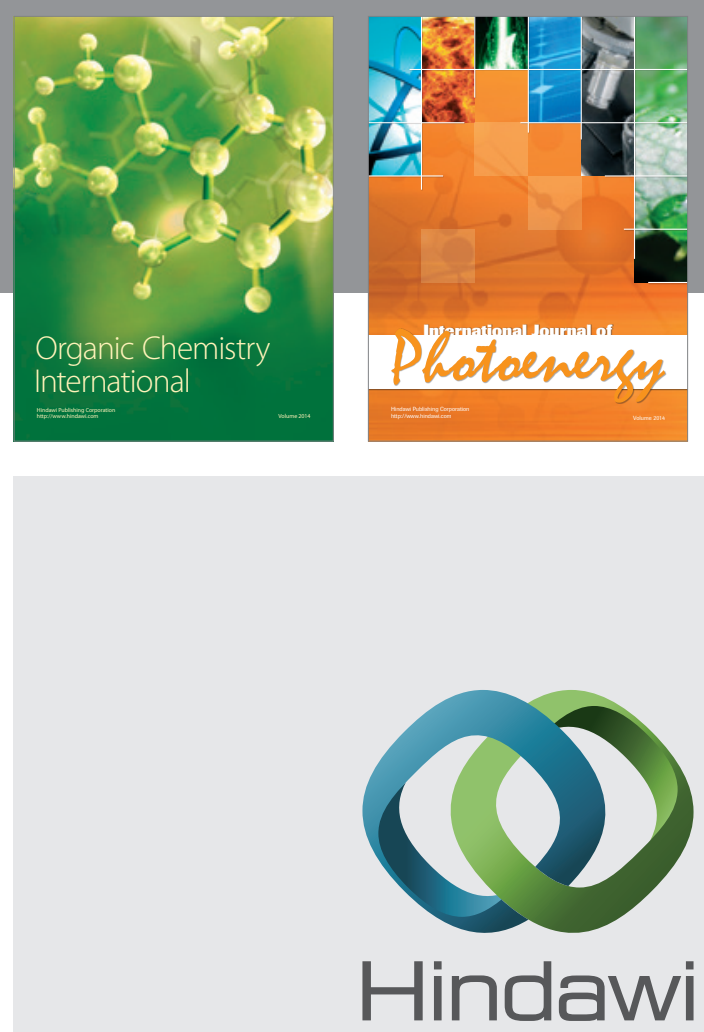

Submit your manuscripts at

http://www.hindawi.com

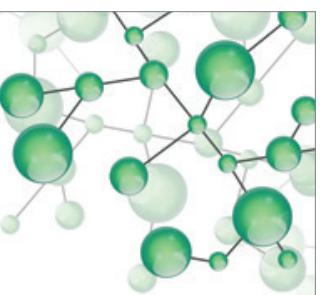

International Journal of

Inorganic Chemistry

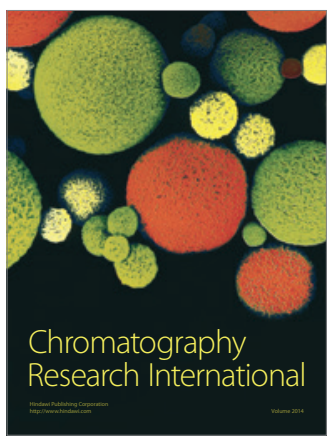

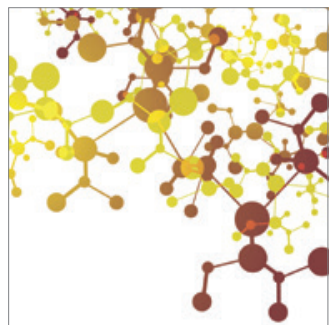

Applied Chemistry
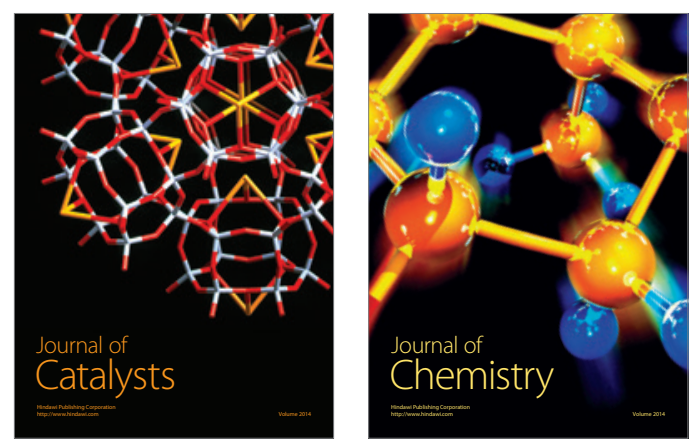
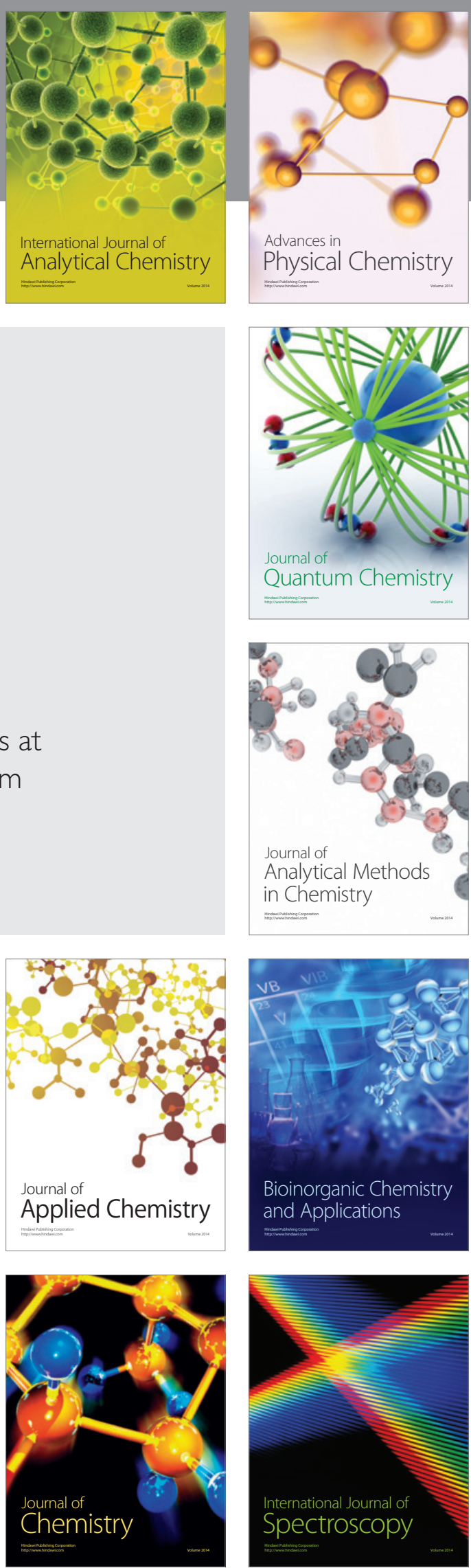